\title{
Determination of the Overshoot Scalar Control Systems with Transfer Zero and Binomial Law of Poles Distribution
}

\author{
Insur Zavdyatovich Ahmetzyanov and Dmitry Nikolayevich Dem'yanov* \\ Kazan Federal University, 420008, Kazan, Kremlyovskaya St., 18, Republic of Tatarstan; \\ insur.zavd23@yahoo.com, demyanovdn@mail.ru
}

\begin{abstract}
Background/Objectives: One of the most popular approaches to the design of automatic control systems is the modal method of controller synthesis. Methods: Its peculiarity is that the requirements for management process quality are formalized by ensuring the desired location on a complex plane of a closed system poles. At that, the introduction of the modal controller makes no effect on the location of transmission zeros. Finding: However, it is known that many transition process quality indicators (e.g., overshoot value) depend not only on the poles but the zeros of the transfer function as well. In work presented here, we analyzed the dependence of overshoot value on the relative position of a dynamic system zeros and poles. Calculation formulas which allow determining the value of a positive or a negative overshoot without the development of transient response are obtained for a scalar system with one zero and the binomial pole distribution law. The dependence of overshoot value on the relative position of the transmission zero and the dynamic system poles is studied. The conditions are formulated, the implementation of which will provide in control system such an overshoot value which does not exceed a predetermined value. Conclusion: The obtained results can be used for further theoretical studies as well as in practice in solving problems of analysis and synthesis of control systems with a modal controller.
\end{abstract}

Keywords: Binomial Distribution Law, Control System, Overshoot, Poles, Transmission Zero, Transient Response

\section{Introduction}

Among currently known approaches to the design of automatic control systems, one of the most famous is the modal method of controller synthesis. Its essence is in the transition of characteristic polynomial roots of a closed system in a certain region of the complex plane by introducing the state feedback or according to an available exit ${ }^{1}$. It is expected that after the implementation of pole desired location in a closed system, a designer will be able to provide the performance you want qualities of management system functioning.

However, as some papers showed, in general, the most important indicators of control system quality (e.g., the overshoot, transient process period, etc.) depend not only on the area of pole localization but also on the location of transfer function zeros ${ }^{2}$. Various authors $\frac{3-6}{}$ examined the relation of zeros and poles location with the peculiarities of transient processes in automatic control systems. However only very general criteria have been currently formulated, they allow estimating only the form of time characteristics and do not allow getting a quantitative assessment of system functioning quality indicators.

As an example, one may consider such indicator as overshoot, which numerically characterizes the maximum deviation of a transient response from a set value. It is evident that its value is one of the most important factors, which should be considered during the study and the design of control systems. At that the known results allow to determine the value of zeros and poles presence or absence of overshoot and also, in some cases, its type (positive or negative). The obtaining of quantitative

${ }^{*}$ Author for correspondence 
estimates becomes possible only as a result of dynamics equation integration, which significantly complicates the practical use of modal control methods.

Thus, the task of detection and research of overshoot value dependence on the arrangement of zeros and poles of a control system with a modal controller is a very actual one.

\section{Problem Setting}

In this paper we consider the control systems, the poles of which are subject to one of distribution laws most frequently used in practice, - a binomial one $\mathrm{e}^{\mathrm{z}}$.

Let the transfer function of a system have the form:

$$
W=k \frac{s+\beta}{(s+\alpha)^{n}} .
$$

It is assumed that $\alpha>0$ (asymptotically stable system), $K \beta>0$ (static gain is positive), $n \geq 2$.

To assess the quality of management processes let's use the overshoot value, which generally may be a positive or a negative one.

The following formula determines the value of a positive overshoot:

$$
\sigma^{+}=\frac{h_{\max }-h_{\infty}}{h_{\infty}} \cdot 100 \% .
$$

Here $h_{\max }$ is the maximum value of the transient response, $h_{\infty}$ is the set value of the transient response (its limit at $t \rightarrow \infty$ ).

The value of a negative over shoot is determined by the following formula:

$$
\sigma^{-}=-\frac{h_{\min }}{h_{\infty}} \cdot 100 \% \text {. }
$$

Here $h_{\min }$ is the minimum value of the transient response.

It should be noted that the negative overshoot is determined only for the cases when a transient response has different signs at various intervals.

It is required to determine the form of dependencies $\sigma^{+}$and $\sigma^{-}$on the values $K, \alpha, \beta$.

\section{Determination of Overshoot Value}

As it was shown in paper -3 cases are possible for the case considered by us depending on the value $\beta$ :
- If $\beta>\alpha$, the transient response has no extreme points. Consequently, the transition process is a monotonous one, and the value of both positive and negative over control is equal to zero.

- If $\beta=\alpha$, the Equation (1) may be written in the following form:

$$
W=k \frac{s+\beta}{(s+\alpha)^{n-1}} \text {. }
$$

In fact, the reduction of zero and one of the transfer function poles takes place. At that the transient response will not have final point's either ${ }^{9}$, the transition process will be the dull one, and the value of both positive and negative overshoot is equal to zero.

It should be noted that in practice this situation of an absolute exact match of zeros and poles is unlikely.

- If $\beta<\alpha$, then the transfer characteristic has a single extreme point. In this case, the existence of a positive or a negative overshoot is possible (the extreme point is single, so the transient process has either one maximum point or one minimum point).

To obtain the value of overshoot let's define an extreme and a set value of the transient response.

The set value of transient response for the system with the transfer function in the form of Equation (1) can be found quickly using the properties of fundamental Laplace transformation.

The following expression determines the picturing of transient response $\mathrm{e}^{\mathrm{10}}$ :

$$
h(s)=\frac{W}{s}=\frac{k(s+\beta)}{s(s+\alpha)^{n}} .
$$

Let's calculate $h_{\infty}$, using the extreme properties of images:

$$
h_{\infty}=\lim _{s \rightarrow 0}[s \cdot h(s)]=\lim _{s \rightarrow 0} \frac{k(s+\beta)}{s(s+\alpha)^{n}}=\frac{k \beta}{\alpha^{n}} .
$$

The largest or smallest value of a transient response is achieved at extreme point, the coordinate of which is determined by the following formula:

$$
t^{*}=\frac{n-1}{\alpha-\beta} \text {. }
$$

Let us find the value of the transient response at extreme point, using the communication between a transient and weight characteristic ${ }^{10}$. 


$$
h\left(t^{*}\right)=\int_{0}^{t^{*}} w(\tau) d \tau
$$

At that it is known that the expression for the weight characteristics in the considered case has the following form:

$$
w(t)=L^{-1}\left\{\frac{k(s+\beta)}{(s+\alpha)^{n}}\right\}=k \frac{t^{n-2} e^{-\alpha t}}{(n-2) !}\left(1+\frac{\beta-\alpha}{n-1} t\right) .
$$

Combining the Equation (5) and (6), we obtain the following:

$$
h\left(t^{*}\right)=\int_{0}^{t^{*}} k \frac{\tau^{n-2} e^{-\alpha \tau}}{(n-2) !}\left(1+\frac{\beta-\alpha}{n-1} \tau\right) d \tau .
$$

Applying to the right part of Equation (7) the integration operation in parts the required number of times, we may obtain an analytical expression for the transient response value at the point of extreme.

If $n=2$, the Equation (7) will have the following form:

$h\left(t^{*}\right)=\int_{0}^{t} k e^{-\alpha \tau}(1+(\beta-\alpha) \tau) d \tau=k\left(\frac{\beta}{\alpha^{2}}+\frac{\alpha-\beta}{\alpha^{2}} \exp \left(\frac{\alpha}{\beta-\alpha}\right)\right)$.

If $n>2$, the Equation (7) will be the following one:

$h\left(t^{*}\right)=\frac{k \beta}{\alpha^{n}}+\frac{k \beta}{\alpha^{n}}\left(\frac{\alpha^{n-1}}{\beta(n-2) !}\left(\frac{n-1}{\alpha-\beta}\right)^{n-2}-\sum_{m=0}^{n-2} \frac{\alpha^{m}}{m !}\left(\frac{n-1}{\alpha-\beta}\right)^{m}\right) \exp \left(\frac{\alpha(n-1)}{\beta-\alpha}\right)$.

To determine the type of extreme point, let's calculate the value of the second derivative, using a well-known relationship between transitional and weight characteristics, as well as by the Expression (6):

$$
\frac{d^{2} h}{d t^{2}}=\frac{d w}{d t}=k \frac{d}{d t}\left(\frac{t^{n-2} e^{-\alpha t}}{(n-2) !}\left(1+\frac{\beta-\alpha}{n-1} t\right)\right) .
$$

If $n=2$, then the Equation (10) will be the following one:

$$
\frac{d^{2} h}{d t^{2}}=k e^{-\alpha t}((\beta-\alpha)(1-\alpha t)-\alpha) .
$$

If $n>2$, then the Equation (10) will be defined by the following formula:

$$
\frac{d^{2} h}{d t^{2}}=\frac{k e^{-\alpha t} t^{n-3}}{(n-2) !}\left((n-2)+(\beta-2 \alpha) t-\frac{\beta-\alpha}{n-1} \alpha t^{2}\right) .
$$

Substituting the Condition (4) into the Expressions (11) and (12), we find the values of the second derivative in an extreme point.
If $n=2$, we obtain the following:

$$
\left.\frac{d^{2} h}{d t^{2}}\right|_{t=t^{*}}=-k(\alpha-\beta) \exp \left(\frac{\alpha}{\beta-\alpha}\right) \text {. }
$$

If $n>2$, we will obtain the following:

$$
\left.\frac{d^{2} h}{d t^{2}}\right|_{t=t^{*}}=-\frac{k}{(n-2) !}\left(\frac{n-1}{\alpha-\beta}\right)^{n-3} \exp \left(-\alpha \frac{n-1}{\alpha-\beta}\right) \text {. }
$$

Since the case $\beta<\alpha$ is considered, then the sign of right-hand sides of obtained equations will be determined solely by the sign of $k$ ratio (all other factors will be positive).

If $k<0$, then the value of the second derivative will be less than zero, and at the considered point the transient response will be the maximum one. If $k<0$, then the value of the second derivative will be positive, in the considered point the transition characteristic will be a minimum one.

Taking into account the previous assumption about the positivity of the product $k \beta$, the obtained results may be formulated somewhat differently. If $\beta>0$, the transient response has a maximum point and a positive overshoot. If $\beta>0$, the transient response has a minimum point and a negative overshoot.

Let's calculate the overshoot value combining the Conditions (2) - (3) with the Expressions (8)-(9). For the case $\alpha>\beta>0$ we obtain the following results.

If $n=2$, then:

$$
\sigma^{+}=\frac{\alpha-\beta}{\beta} \exp \left(\frac{\alpha}{\beta-\alpha}\right) \cdot 100 \% \text {. }
$$

If $n>2$, then:

$$
\sigma^{+}=\frac{1}{\beta}\left(\alpha-\beta+\sum_{k=1}^{n-2} \frac{k \alpha^{n-k-1}}{(n-k-1) !}\left(\frac{n-1}{\alpha-\beta}\right)^{n-k-2}\right) \exp \left(\frac{\alpha(n-1)}{\beta-\alpha}\right) \cdot 100 \% .
$$

At $\beta<0$ the value of negative overshoot will be determined by the following expressions.

$$
\text { If } n=2 \text {, then: }
$$

$$
\sigma^{-}=\left(\frac{\beta-\alpha}{\beta} \exp \left(\frac{\alpha}{\beta-\alpha}\right)-1\right) \cdot 100 \% \text {. }
$$

If $n>2$, then:

$$
\sigma^{-}=\left(\frac{1}{\beta}\left(\beta-\alpha-\sum_{k=1}^{n-2} \frac{k \alpha^{n-k-1}}{(n-k-1) !}\left(\frac{n-1}{\alpha-\beta}\right)^{n-k-2}\right) \exp \left(\frac{\alpha(n-1)}{\beta-\alpha}\right)-1\right) \cdot 100 \% \text {. }
$$


Let us analyze the nature of overshoot value dependence from the value $\alpha$ at set $\beta$ ((the physical meaning in this case is explained by the fact that at the modal control the pole location may be set arbitrarily, and the feedback has no effect on the location of zeroes).

If $\alpha>\beta>0$, the transient response has only a positive overshoot, defined by the Relations (13) and (14). Differentiating them according to $\alpha$, we obtain the following dependencies.

If $n=2$, then:

$$
\frac{\partial \sigma^{+}}{\partial \alpha}=\frac{\alpha}{\beta(\alpha-\beta)} \exp \left(\frac{\alpha}{\beta-\alpha}\right) \cdot 100 \% \text {. }
$$

If $n>2$, then:

$$
\frac{\partial \sigma^{+}}{\partial \alpha}=\frac{\alpha^{n-1}}{\beta(n-1) !}\left(\frac{n-1}{\alpha-\beta}\right)^{n-1} \exp \left(\frac{\alpha(n-1)}{\beta-\alpha}\right) \cdot 100 \% \text {. }
$$

If the provided limitations are performed, the functions defined by the Expressions (17) - (18) will always be above zero. Consequently, the dependence $\sigma^{+}(\alpha)$ is the increasing function - the more the value of a pole, the larger the value of a positive overshoots.

If $\alpha>\beta>0$, the transient response has only a negative overshoot, defined by the Relations (15) and (16). Differentiating them by $\alpha$, we obtain the following dependencies.

If $n=2$, then:

$$
\frac{\partial \sigma^{-}}{\partial \alpha}=-\frac{\alpha}{\beta(\alpha-\beta)} \exp \left(\frac{\alpha}{\beta-\alpha}\right) \cdot 100 \% \text {. }
$$

If $n>2$, then:

$$
\frac{\partial \sigma^{-}}{\partial \alpha}=-\frac{\alpha^{n-1}}{\beta(n-1) !}\left(\frac{n-1}{\alpha-\beta}\right)^{n-1} \exp \left(\frac{\alpha(n-1)}{\beta-\alpha}\right) \cdot 100 \% \text {. }
$$

When you perform abovementioned limitations, the functions defined by the Expressions (19) - (20) will always be larger than zero. Consequently, the dependence $\sigma(\alpha)$ is the increasing function - the more the value of a pole, the more the value of a negative overshoot.

Thus, for a fixed value of transfer zero at the removal of location point of a closed system pole from the coordinate beginning the value of both positive and negative overshoot will be increased. Therefore, if the requirements for the designed control system have a set limit value of overshoot, the performance of such a system (defined by value $\alpha$ ) cannot exceed some minimum value.
The maximum value $\alpha$, providing the allowable value of overshoot $\sigma^{+}$or $\sigma$, may be determined from the Relations (13)-(16). To do this, we have to present them as non-linear equations with respect to $\alpha$ and to solve it by any of the known numerical methods.

\section{Examples}

To illustrate the usefulness of obtained results, let's consider two methodological examples.

- Let the transfer function of an automatic control system is defined by the following formula:

$$
W=\frac{2500(s+4)}{(s+10)^{4}} \text {. }
$$

It is required to determine the nature of transition process and the value of overshoot.

The control system has one zero, $\beta=4, \alpha=10, n=4$.

Since $\alpha>\beta$, the transition process will have an overshoot. Since $\beta>0$, the overshoot will be positive.

In order to determine the value of overshoot let's use the Formula (14):

$$
\sigma^{+}=\frac{1}{4}\left(6+\sum_{k=1}^{2} \frac{k \cdot 10^{3-k}}{(3-k) !}\left(\frac{3}{6}\right)^{2-k}\right) \cdot \exp \left(-\frac{30}{6}\right) \cdot 100 \% \approx 8,6 \%
$$

The correctness of obtained results is confirmed by the results of numerical simulation, conducted in the system of computer mathematics MATLAB. The schedule of the transition process for the control system with a given transfer function is shown on Figure 1.

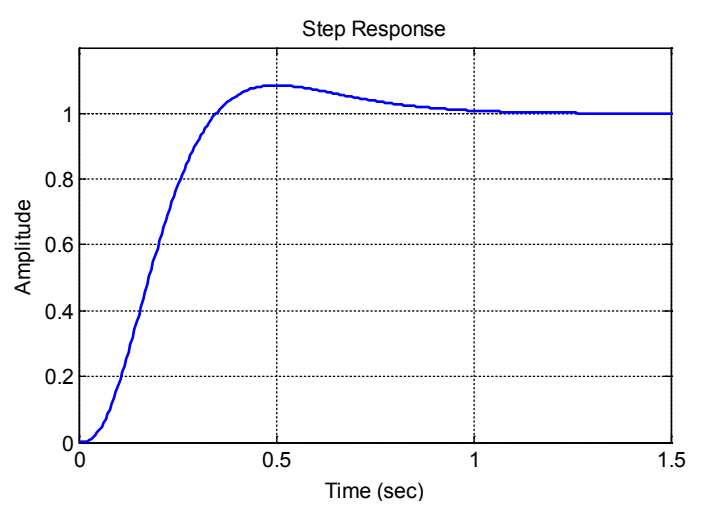

Figure1. The schedule of transient response for Example 1.

- Let the dynamics of the control object be described by a linear differential equation of the 
second order. At that the object has one transmission zero, $\beta=5$.

It is planned to synthesize the controller according to a state by the method of modal control, the desired location of the roots for characteristic equation is subjected to binomial distribution law.

It is required to determine the values of $\alpha$ at which overshoot value in the projected control system will not exceed $10 \%$.

Since $\beta>0$, then the closed system either would have a positive overshoot (if $\alpha=5$ ), or will not have any overshoot (if $\alpha \leq 5$ ).

In order to determine the maximum value $\alpha$ let's use the following Formula (13):

$$
10=\frac{\alpha-5}{5} \cdot \exp \left(\frac{\alpha}{5-\alpha}\right) \cdot 100 .
$$

Having solved the nonlinear equation by the method of simple iterations, we will get $\alpha_{0} \approx 9,32$. Thus, the projected control system will have an allowable overshoot only if $\alpha \leq 9,32$.

Figure 2 shows the graphs of the closed system transient response at different values of $\alpha$, obtained using the MATLAB computer mathematics system, fully confirming the validity of the obtained result.

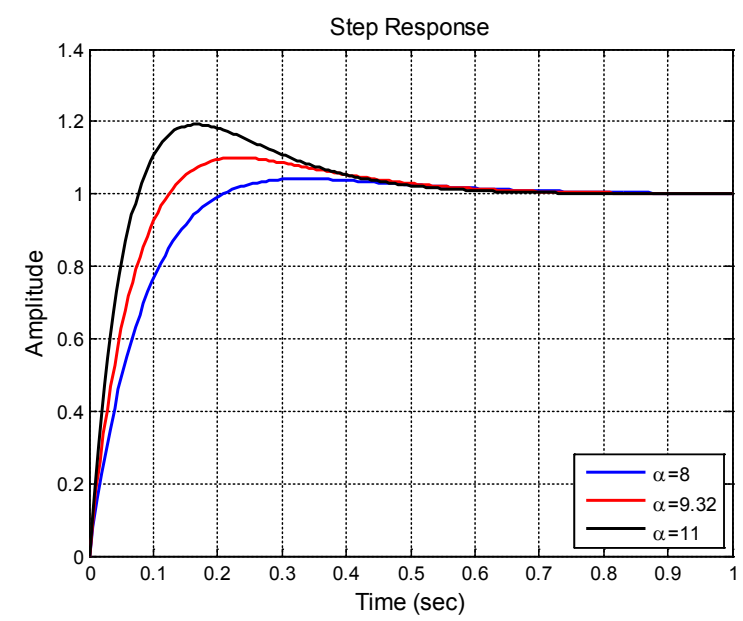

Figure2. Transient characteristic graphs for Example 2.

Thus, the obtained analytical expressions can be used to solve the problems of control system analysis and synthesis with set quality characteristics.

\section{Conclusions}

By obtained relations, one may conclude that in the control systems with a binomial pole distribution law the value of overshoot essentially depends on the location of the transmission zero. If zero is located on a complex plane to the right of the poles, the transition process in such a system is characterized by the overshoot, the value of which will be increased at distance increase between zero and poles. If the transmission zero is located in the right half plane, the overshoot will be negative, and its value will be increased with zero-distancing from coordinate origin.

\section{Summary}

Thus, the present study revealed the dependence of value and nature of overshoot in the systems of automatic control with a binomial law of pole distribution on the complex plane of the transmission zero. The analytical ratios are obtained, which allow to estimate the value of overshoot with a high degree of accuracy and to determine allowable values of the closed system poles according to a set regulation. The obtained results can be used in practice at the design of modal controllers for control objects with a transmission zero.

\section{Conflict of Interest}

The authors acknowledge that the presented data do not contain any conflict of interest.

\section{Acknowledgements}

The work is performed according to the Russian Government Program of Competitive Growth of Kazan Federal University.

This work was supported by the Russian Foundation for Basic Research (Grant No. 16-38-00042).

\section{References}

1. Olio Model One: Why the Craft Brew of Smart watches Actually Goes Flat. Available from: http://readwrite.com/2015/04/01/ olio-model-one-smartwatch-craft-beer-flat/

2. Graham C, Stefan F, Mario E. Control system design. Upper Saddle River, New Jersey: Prentice-Hall; 2001 Mar.

3. Smagina EM. The issues of linear multivariate object analysis using the concept of the system zero. Tomsk: Publishing house of Tomsk University; 1990.

4. Kobayashi T. Zeros and design of control systems for distributed parameter systems. International Journal of Systems Science.1992; 23(9):1507-15. 
5. Jambhekar ND, Dhawale CA. bit level key agreement and exchange protocol for digital image steganographic. Indian Journal of Science and Technology. 2015 Jul; 8(15):1-7.

6. Ramírez HS, Rodríguez CG, Romero JC, Juárez AL. Algebraic identification and estimation methods in feedback control systems. Wilky; 2014 Mar. p. 390.

7. Antsaklis PJ, Michel AN. Linear systems. New York: McGraw-Hill; 1997.

8. Demyanov DN. Impact of transmission zeros spectrum on the nature of transient process in the scalar control systems with binomial law of poles distribution. Global Journal of Pure and Applied Mathematics. 2015 Jan; 11(6):5089-98.

9. Kalaiselvi B. Design of smart positioner for a control valve to optimize backlash problem. Indian Journal of Science and Technology. 2015 Nov; 8(32):1-7.

10. Baillieul J, Samad T. Encyclopedia of systems and control. London: Springer; 2015. 